\title{
Adsorption and Desorption Dynamics of Sodium Dodecyl Sulfate at the Octadecylsilane Layer on the Pore Surface of a Mesoporous Silica Film Observed in-situ by Optical Waveguide Spectroscopy
}

\author{
Akira Yamaguchi, ${ }^{* 1, * 2 \dagger}$ Hiroyuki Arafune, ${ }^{* 3}$ Kazuhiro HotTa, ${ }^{* 3}$ Tetsuji ItoH, ${ }^{* 4}$ and \\ Norio TeramaE*3† \\ *1 College of Science, Ibaraki University, 2-1-1 Bunkyo, Mito, Ibaraki 310-8512, Japan \\ *2 Frontier Research Center for Applied Atomic Sciences, Ibaraki University, Tokai, Ibaraki 319-1106, Japan \\ *3 Department of Chemistry, Graduate School of Science, Tohoku University, Aoba, Sendai 980-8578, Japan \\ *4 Research Center for Compact Chemical System, National Institute of Advanced Industrial Science and \\ Technology (AIST), 4-2-1 Nigatake, Miyagino, Sendai 983-8551, Japan
}

\begin{abstract}
The purpose of this study is to apply optical waveguide (OWG) spectroscopy to observe adsorption and desorption dynamics occurring in a surfactant-templated mesoporous silica film. For that purpose, a mesoporous silica (MS) film with open accessible pores (pore diameter, $c a .6 \mathrm{~nm}$ ) was formed on an aluminum (Al) layer deposited on a glass substrate, and the pore surface of the MS film was modified with octadecylsilane (ODS). The resulting ODS-modified MS (ODS-MS) and Al multilayer film showed a clear waveguide coupling dip in the reflection spectrum. The position of the waveguide coupling dip was red-shifted as the amount of sodium dodecyl sulfate within the ODS-MS layer increases. These results indicate the usefulness of OWG spectroscopy for the study of adsorption/desorption dynamics occurring in MS materials.
\end{abstract}

(Received February 7, 2011; Accepted April 17, 2011; Published June 10, 2011)

\section{Introduction}

In the past two decades, inorganic nanoporous materials with uniform and ordered pore structures have been widely applied in various fields due to their large surface area, molecular sieve effect, and specific physical and chemical properties that depend on the pore size. ${ }^{1-4}$ Adsorption and desorption of solute molecules are the key step to incorporate functional groups into the pores and to use the functions inside the pores. Accordingly, adsorption/desorption dynamics of molecules in the inorganic nanoporous materials has been studied by in-situ observation techniques utilizing a quartz crystal microbalance (QCM) $)^{5,6}$ and optical microscopes. ${ }^{7,8}$ Recently, light propagation in a nanoporous film has been used to monitor various processes occurring inside the pores by means of Fabry-Perot fringes,, 910 photonic band gap (PBG), ${ }^{11,12}$ and waveguide mode. ${ }^{13-17}$ Since these optical properties are sensitive to the refractive index change of the nanoporous film induced by adsorption/desorption of molecules inside the pores, changes in such optical properties can be utilized for in-situ observation of adsorption/desorption dynamics of a wide variety of molecules with and without strong light absorption and emission properties.

Optical waveguide (OWG) spectroscopy is based on the OWG mode excited in a nanoporous film on a metal thin film, which appears as a sharp attenuation dip in a reflection spectrum (reflectivity $v s$. incident angle or wavelength) from a

† To whom correspondence should be addressed.

E-mail: yakira@mx.ibaraki.ac.jp; teramae@m.tains.tohoku.ac.jp nanoporous/metal multilayer film. ${ }^{13-18}$ The position of the attenuation dip shifts depending on the change of the real part of the complex refractive index of a nanoporous film, whereas the reflectivity of the attenuation dip changes depending on the imaginary part of the complex refractive index of a nanoporous film. ${ }^{17}$ Thus, OWG spectroscopy provides valuable information on the amount and the extinction coefficient of an adsorbate inside nanopores. In addition, since the OWG mode can be excited by both p- and s-polarized light, ${ }^{19}$ the optical anisotropy of a nanoporous film can be analyzed by OWG spectroscopy. Accordingly, OWG spectroscopy has been regarded as a powerful tool for in-situ observation of adsorption/desorption dynamics occurring in a nanoporous film, and it has been applied to several inorganic nanoporous film systems such as a porous anodic alumina (PAA) film, ${ }^{15-17}$ and a nanoporous $\mathrm{TiO}_{2}$ film composed of aggregated colloidal $\mathrm{TiO}_{2}$ particles. ${ }^{13,14}$

Mesoporous silica (MS) materials have uniform and ordered mesopores; the pore diameter can be continuously tuned from ca. $2 \mathrm{~nm}$ to $c a .20 \mathrm{~nm}$ by choosing appropriate surfactants and precursor compositions. ${ }^{1,20,21}$ The tunable structural characteristics of MS materials are advantageous to optimize the refractive index of the MS layer that acts as a sensing layer in OWG spectroscopy. ${ }^{17}$ In addition, the improved enzymatic activity obtained by incorporation of enzymes into the pores of MS materials ${ }^{3}$ stimulates us to examine the adsorption and desorption behaviors of molecules in the pores of MS materials. Accordingly, an octadecylsilyl (ODS)-modified MS (ODS-MS) layer was formed by using triblock copolymer surfactants as a template, and this ODS-MS layer was utilized as a sensing layer in OWG spectroscopy to examine adsorption and desorption 
dynamics of sodium dodecyl sulfate (SDS). The hydrolysis of silica-framework is one of critical problems for the usage of a MS film in an aqueous solution, so its suppression techniques have been extensively studied. Such techniques include co-condensation of silica, addition of secondary metal oxides, and surface modification. ${ }^{22-25}$ The ODS layer of ODS-MS can also work to suppress the silica hydrolysis. The effect of the structural and optical properties of the ODS-MS layer on the excitation of the OWG mode was examined by forming ODS-MS on a thin aluminum (Al) layer, and the features of OWG spectroscopy of the ODS-MS/Al multilayer film were examined by observing the adsorption/desorption dynamics of SDS.

\section{Experimental}

\section{Reagents and chemicals}

A rectangular cover glass slip $(24 \times 45 \times 0.3 \mathrm{~mm}$, Matsunami Glass Ind., Ltd., Osaka, Japan) was used for the fabrication of the ODS-MS/Al multilayer film. Al wire (99.99\%; Nilaco Co., Tokyo, Japan) was used for the thermal deposition of Al films. Milli-Q water was used for all experiments. Pluronic F127 (F127: $\mathrm{PEO}_{106} \mathrm{PPO}_{70} \mathrm{PEO}_{106}$ ), which was a non-ionic triblock copolymer consisting of polyethyleneoxide (PEO) and polypropyleneoxide (PPO), was purchased from Sigma-Aldrich (Tokyo, Japan). Other chemicals were purchased from Wako Pure Chemical Industries, Ltd. (Osaka, Japan).

\section{Preparation of ODS-MS film}

A precursor solution for the MS layer was prepared according to the literature ${ }^{26}$ with some modifications. A mixture of F127 $(1.38 \mathrm{~g}), 1 \mathrm{M}$ hydrochloric acid solution $(0.30 \mathrm{~g})$, ethanol $(26.49 \mathrm{~g})$, and water $(2.07 \mathrm{~g})$ was stirred for $1 \mathrm{~h}$ at $30^{\circ} \mathrm{C}$. Then, tetraethoxysilane (TEOS, $3.0 \mathrm{~g}$ ) was added to the mixture and the mixed solution was stirred for $2 \mathrm{~h}$ at $30^{\circ} \mathrm{C}$ to give the precursor solution.

The glass substrate was sonicated in acetone, methanol, and then Milli-Q water for 5 min each. Then it was immersed in concentrated nitric acid and boiled for 75 min to remove any organic species adsorbed on the substrate surface. Next, its surface was etched by immersing it in a $10 \mathrm{wt} \%$ hydrofluoric acid solution for $20 \mathrm{~s}$. After the glass substrate was rinsed with water and dried under a nitrogen gas stream, an Al layer $(17 \mathrm{~nm})$ was deposited on one side of the glass substrate using a Ulvac Model VPC-1100 vacuum deposition system. Then, MS layers were formed on both sides of the Al-deposited glass substrate from the precursor solution by dip-coating at a withdrawal rate of $4 \mathrm{~mm} / \mathrm{s}$. After the dip-coating, the Al-deposited glass substrate with as-synthesized MS layers was heated at $70^{\circ} \mathrm{C}$ overnight. The MS layer on the Al-deposited side of the substrate was utilized in the measurements of adsorption/desorption dynamics.

The Al-deposited glass substrate with the as-synthesized MS layers was immersed in $30 \mathrm{ml}$ of dry toluene containing $0.91 \mathrm{~g}$ of octadecyldimethylchlorosilane, and heated under reflux for $24 \mathrm{~h}$. After reflux, the substrate was rinsed with dry toluene and dry ethanol several times, and dried under vacuum for $1 \mathrm{~h}$. For end-capping of residual surface silanol groups, the Al-deposited glass substrate with ODS-modified MS (ODS-MS) layers was immersed in $29.4 \mathrm{ml}$ of dry toluene and $0.6 \mathrm{ml}$ of trimethylethoxysilane, and heated under reflux for $24 \mathrm{~h}$. The substrate was then rinsed and dried again according to the procedures described above to form the sample substrate with the ODS-MS/Al multilayer film on the glass substrate. During

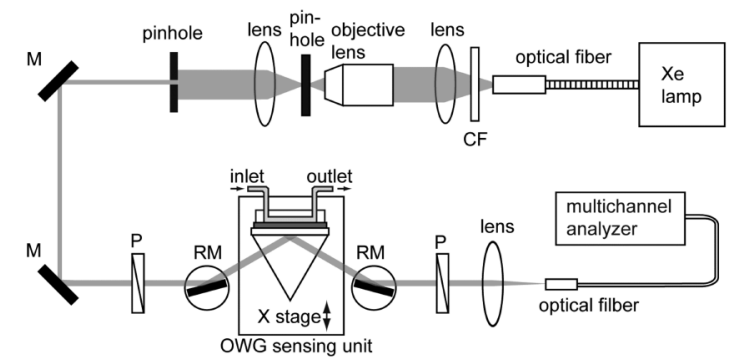

Experimental setup for OWG spectroscopy

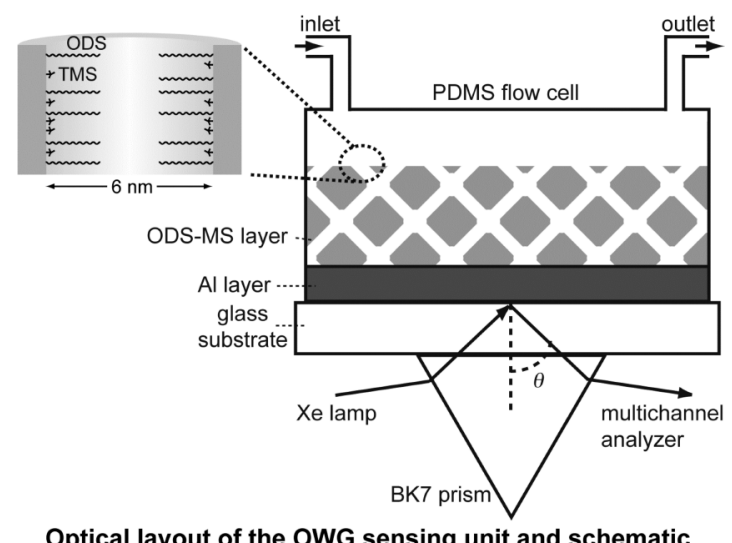

Optical layout of the OWG sensing unit and schematic of the ODS-MS/AI multilayer film on a glass substrate

Fig. 1 Experimental setup for OWG spectroscopy (upper) and the expanded view of the OWG sensing unit with schematic of the ODS-MS/Al multilayer film (lower). CF, cold filter; M, plane mirror; $\mathrm{P}$, polarizer; $\mathrm{RM}$, rotatable plane mirror.

the above modification and drying procedures, the template surfactants were replaced by ODS and trimethylsilyl (TMS) groups using the reported modification methods. ${ }^{27,28}$

For the characterization of the optical properties of the ODS-MS layer, a reference substrate was prepared by forming ODS-MS layers on a glass substrate without the Al layer. Formation of MS layers and modification with ODS and TMS groups were performed by the same manner as described above. Similar to the reference substrate, two glass substrates were prepared by forming as-synthesized MS layers and calcined MS layers for comparison. Since the ODS-MS layers are formed on the both sides of substrates by dip-coating, the reference substrate has a layered structure of ODS-MS/glass/ODS-MS and the sample substrate, ODS-MS/Al/glass/ODS-MS. Hereafter, the reference substrate is defined as ODS-MS/glass and the sample substrate as ODS-MS/Al/glass for the sake of simplicity.

The pore structure of the ODS-MS layer was confirmed by field emission scanning electron microscopy (FE-SEM; HITACHI, S-4800) and by an X-ray diffraction (XRD) measurement. The XRD pattern of the ODS-MS layer was recorded on a MAC Science/Bruker M21X with $\mathrm{Cu} \mathrm{K}$ radiation. The diffraction pattern was recorded in the $2 \theta$ range of $0.5^{\circ}-10^{\circ}$, at $0.01^{\circ} 2 \theta$ step size and $2 \mathrm{~s}$ step time, running at a voltage of $45 \mathrm{kV}$.

\section{OWG spectroscopy}

Reflection spectra (reflectivity vs. wavelength of incident light) of the ODS-MS/Al multilayer film were measured in the Kretschmann configuration. ${ }^{18}$ The experimental setup for the OWG spectroscopy is shown in Fig. 1. A Xe lamp (Hamamatsu 
Photonics; L8254, $150 \mathrm{~W}$ ) was used as a white light source. White light from the Xe lamp was collimated by a spatial filter assembly composed of a $25-\mu \mathrm{m}$ pinhole, a $10 \times$ objective lens, and a collimating lens $(f=25.2 \mathrm{~mm})$. The polarization of the collimated white light was set to p-polarization by using a polarizer (Sigma Koki Co., Ltd.; SPF), and the p-polarized light beam was directed onto the sample substrate attached to a BK7 equilateral dispersing prism via an index matching fluid (Cargille; immersion oil No. 16242). The optical properties of the ODS-MS layer between the BK7 prism and the cover glass slip of sample substrate were negligible in the measurements of OWG modes because of its thin thickness. A polarizer was placed between the BK 7 prism and a multichannel analyzer (Hamamatsu Photonics; PMA-11) to detect only the p-polarized component of reflected light. The reflectivity of the ODS-MS/Al multilayer film was normalized using the intensity of reflection light observed for a bare glass substrate attached to the BK7 prism. It should be noted that the incident angle at $632.8 \mathrm{~nm}$ is designated as a representative incident angle in the measurements of reflection spectra. A fluidic channel (channel width, $3 \mathrm{~mm}$; channel depth, $0.2 \mathrm{~mm}$ ) was fabricated in polydimethylsiloxane (PDMS) substrate, and the PDMS-channel substrate was placed on the ODS-MS/Al multilayer film. A syringe pump (Harvard Apparatus; Model 11 plus) was used to deliver the sample solution into the fluidic channel and the OWG spectroscopy measurements were performed using continuous solution flow at $100 \mu 1 \mathrm{~min}^{-1}$.

\section{Optical parameters for Fresnel calculations}

Fresnel calculations were carried out to discuss the reflection spectra of the ODS-MS multilayer film using the "Mathematica" version 5.2 software (Wolfram Research, Inc., Champaign, IL). Details of the calculations were described in our previous literature. ${ }^{17}$ For the Fresnel calculations, refractive indices of $\operatorname{BK} 7\left(n_{\mathrm{BK} 7}=1.50+4330 / \lambda^{2}\right)$ and water $\left(n_{\mathrm{W}}=1.32+4340 / \lambda^{2}-\right.$ $\left.1.83 \times 10^{8} / \lambda^{4}\right)$ were used. ${ }^{17}$ The complex refractive index of the $\mathrm{Al}$ layer was experimentally determined by measuring reflection spectra of an Al film deposited on a glass substrate and fitting them by the Fresnel calculations. ${ }^{17}$ The refractive index of the glass $\left(n_{\mathrm{g}}=1.51+4320 / \lambda^{2}\right)$ substrate was estimated by the best fit of the Fresnel calculated results to the optical transmission spectrum of the glass substrate. The refractive index of ethanol $\left(n_{\mathrm{EtOH}}=1.35+3553 / \lambda^{2}\right)$ was estimated by the best fit of the Fresnel calculated results to the optical reflection spectrum obtained for the glass/ethanol interface. The refractive index of the ODS-MS layer on the glass substrate was estimated by analyzing the optical interference fringes observed in its light transmission spectrum (see Fig. 4).

\section{Results and Discussion}

\section{Characterization of ODS-MS layer}

Figure 2 shows typical SEM images of the sample substrate (ODS-MS/Al/glass). The SEM top view shows the presence of mesochannels on the top surface of the ODS-MS layer. On the other hand, a relatively ordered pore structure is recognized for the SEM cross-sectional view after cleavage of the sample substrate. Figure 3 shows XRD patterns of the sample substrate and reference substrate (ODS-MS/glass). In both XRD patterns, two diffraction peaks are recognized at around $0.72^{\circ}$ and $1.33^{\circ}$, which respectively correspond to $d$ values of 12.2 and $6.6 \mathrm{~nm}$. The coincidence of XRD patterns shown in Fig. 3 indicates formation of an equivalent mesostructure for both ODS-MS layers. The SEM images and XRD patterns shown in Figs. 2
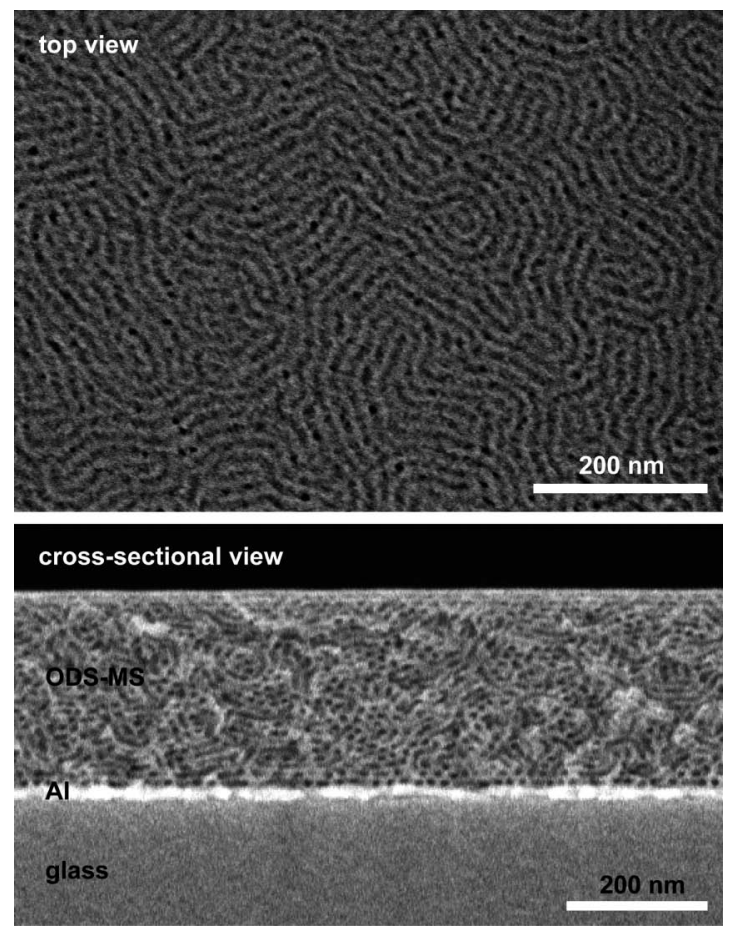

Fig. 2 SEM images of the ODS-MS/Al multilayer film on a glass substrate.

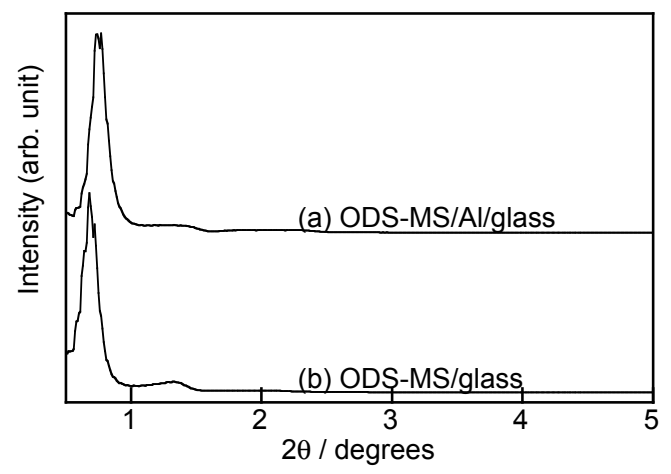

Fig. 3 XRD patterns of (a) the sample substrate (ODS-MS/Al/glass) and (b) the reference substrate (ODS-MS/glass).

and 3 are equivalent to those of the MS film with a layered structure which was formed by dip-coating using a precursor solution containing F127 as a template surfactant. ${ }^{26}$ The pore diameter estimated from the SEM images was $c a .6 \mathrm{~nm}$.

For the estimation of the refractive index of the ODS-MS layer, optical transmission spectrum of the reference substrate was measured. As shown in Fig. 4(a), the typical reference substrate shows a clear optical interference pattern in its light transmission spectrum, and the interference pattern can be well-fitted to the three phase (ODS-MS/glass/ODS-MS) Fresnel calculations. The well-fitted result indicates the optically uniform thickness of the ODS-MS layer. The Fresnel fitting analysis of the interference pattern provides the thickness of the ODS-MS layer as $370 \mathrm{~nm}$, which agrees well with the one evaluated from the SEM image. The refractive index of the ODS-MS layer was also obtained by the Fresnel fitting analysis. Figure 4(b) shows the dispersion of the refractive indices of the 

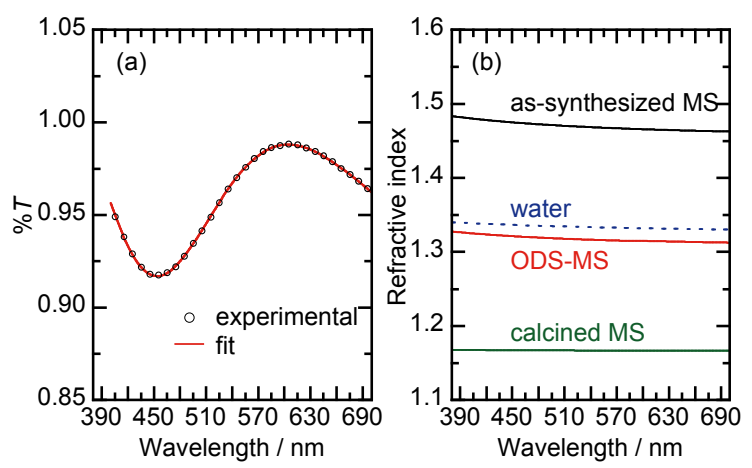

Fig. 4 (a) Optical transmittance spectrum of the reference substrate (ODS-MS/glass). (b) Dispersion of refractive indices of as-synthesized MS, calcined MS, and ODS-MS layers on glass substrates, together with the refractive index dispersion of water.

three types of MS layers on glass substrates together with the refractive index dispersion of water. The refractive indices are higher in the order of as-synthesized MS, ODS-MS, and calcined MS layers on glass substrates. The as-synthesized MS layer has densely-packed template surfactants inside the silica mesopores. On the other hand, the calcined MS layer has empty silica mesopores due to the removal of the template surfactants by calcination. The pore surface of the ODS-MS layer are modified by the ODS and TMS groups but these groups are not fully packed on the pore surface. The structural characteristics of these MS layers are well reflected in Fig. 4(b).

Since the sample substrate showed a mesostructure equivalent to that of the reference substrate (Fig. 3), the dispersion of the refractive index of the ODS-MS layer shown in Fig. 4(b) was used to discuss the OWG mode for the ODS-MS/Al multilayer film of the sample substrate.

\section{OWG mode for the ODS-MS/Al multilayer film}

Figure 5(a) shows the reflection spectra of the ODS-MS/Al multilayer film measured in contact with ethanol. Clear waveguide coupling dips corresponding to the $\mathrm{TM}_{1}$ waveguide coupling mode can be recognized when the incident angle of light is larger than $64.0^{\circ}$ which is above the critical angle. ${ }^{17}$ The coupling wavelength $\left(\lambda_{\text {owG }}\right.$ : wavelength at minimum reflectivity in the waveguide coupling dip) is blue-shifted as the incident angle increases. These features of waveguide coupling dips can be well simulated by five phase Fresnel calculations (BK7/glass/Al/ODS-MS/ethanol) by assuming that the pores of ODS-MS are filled with ethanol molecules (Fig. 5(b)). The porosity of ODS-MS was evaluated as $31 \%$ from the fitting results by Fresnel calculations, and this value is comparable with that found in MS materials prepared by Pluronic F127 as the template surfactant. ${ }^{29}$

On the other hand, no waveguide coupling dip was observed when reflection spectra of the ODS-MS/Al multilayer film were measured in contact with water as typically shown in Fig. 6(a). This phenomenum can be explained by the refractive index of the ODS-MS layer being smaller than that of the water phase (Fig. 4(b)). Since the ODS-MS layer rejects the penetration of water molecules into the silica mesopores modified with hydrophobic ODS groups, the refractive index of the ODS-MS layer in contact with water is comparable with that found in air and is lower than that of the water phase. On the basis of Fresnel calculations, the waveguide coupling mode in the ODS-MS/Al multilayer film cannot be excited when the
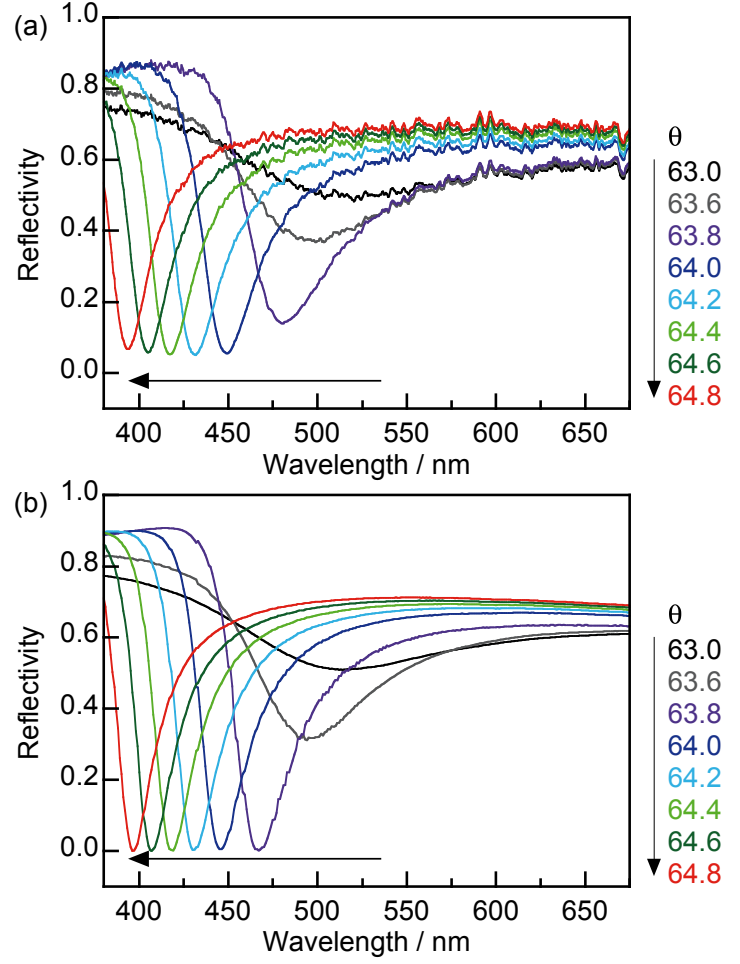

Fig. 5 (a) Reflection spectra of the ODS-MS/Al multilayer film in contact with ethanol measured at a series of incident angles. The thicknesses of the ODS-MS and Al layers are 370 and $17 \mathrm{~nm}$, respectively. (b) Simulated reflection spectra produced by five phase (BK7/glass/Al/ODS-MS/ethanol) Fresnel calculations.

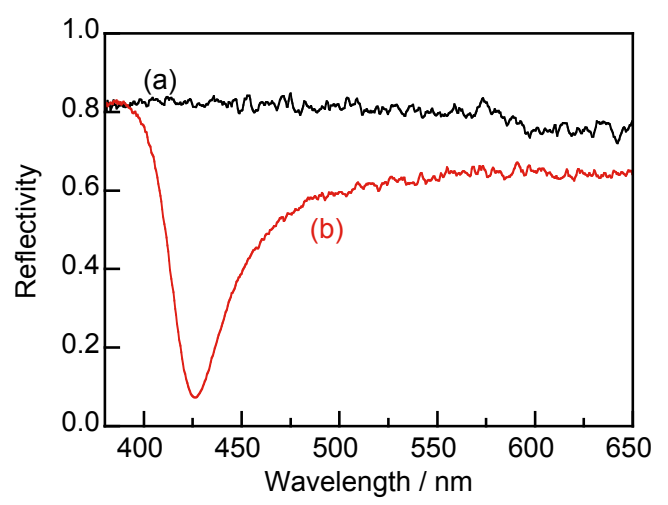

Fig. 6 Reflection spectra of the ODS-MS/Al multilayer film in contact with (a) water and (b) a 99:1 v/v water/ethanol mixture containing $0.4 \mathrm{M} \mathrm{NaCl}$. The thicknesses of the ODS-MS and Al layers are 360 and $17 \mathrm{~nm}$, respectively.

refractive index of the ODS-MS layer is lower than that of the solution phase. Accordingly, we conclude that the penetration of solvent molecules into the silica mesopores is necessary to increase the refractive index of the ODS-MS layer for the excitation of the waveguide coupling mode.

As shown in Fig. 6, a clear waveguide coupling dip can be obtained when the ODS-MS layer is in contact with a 99:1 v/v water/ethanol mixture containing $0.4 \mathrm{M} \mathrm{NaCl}$, indicating that a mixture of ethanol and water can penetrate into the ODS-modified silica mesopores. 

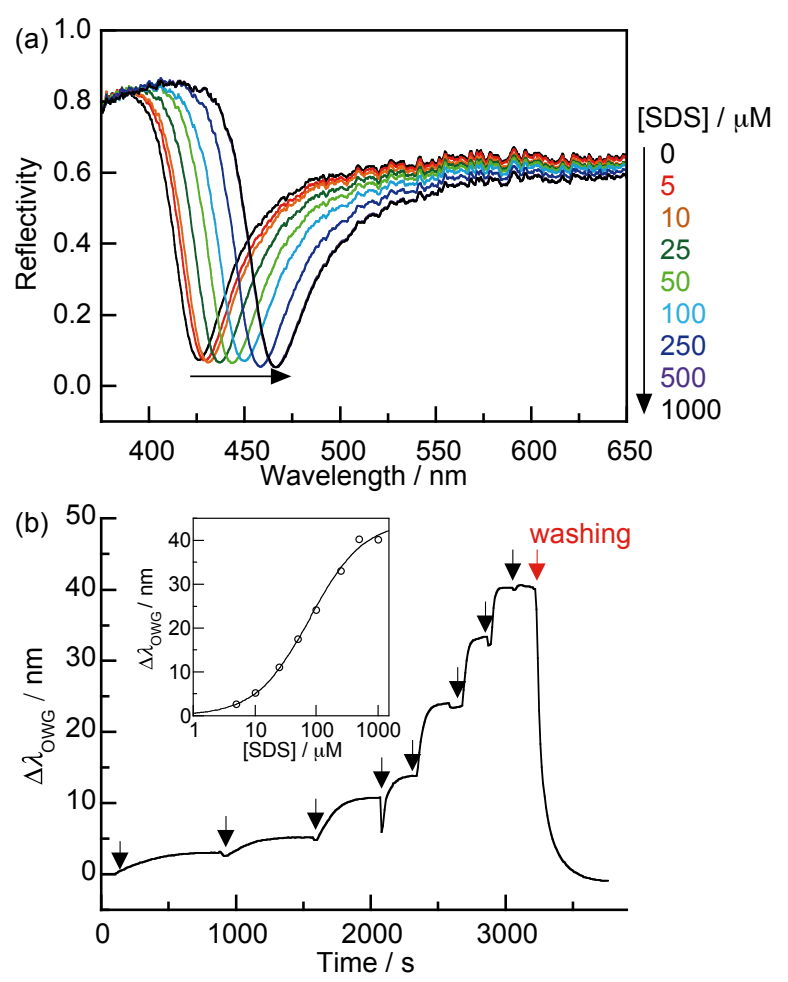

Fig. 7 (a) Reflection spectra of the ODS-MS/Al multilayer film in contact with 99:1 v/v water/ethanol mixtures containing different concentrations of SDS. (b) Time-course shift of the waveguide coupling wavelength $(\Delta \lambda)$ upon addition of SDS. Black arrows indicate the injection of solutions containing different concentrations of SDS. The red arrow indicates the injection of a water/ethanol mixture for washing. The inset in (b) shows the adsorption isotherm of SDS.

\section{Adsorption/desorption behaviors of SDS}

Adsorption behaviors of SDS have been studied on the hydrophobic surfaces of porous silica particles, ${ }^{30}$ and one would expect that SDS interacts with the ODS layer of the ODS-MS/Al multilayer film. To examine adsorption/desorption dynamics of SDS, one must keep the refractive index of a sample solution containing SDS at a constant value irrespective of the SDS concentration. Accordingly, the excess amount of $\mathrm{NaCl}$ was added to the sample solution $([\mathrm{NaCl}]=0.4 \mathrm{M})$ compared with the SDS concentration $(0-1.0 \mathrm{mM})$. As shown in Fig. 7(a), parallel red-shift of the coupling wavelength, $\lambda_{\text {owG }}$, was observed with increasing the concentration of a SDS solution. Figure 7(b) shows the time-course shift of the coupling wavelength observed by changing the concentration of the SDS solution. When each SDS solution with different concentrations was injected into the flow cell (indicated by black arrow), the coupling wavelength shows a gradual red-shift and reaches a plateau within a few hundred seconds. This red-shift of the coupling wavelength can be ascribed to the increase in the refractive index of the ODS-MS layer accompanied by adsorption of SDS on the ODS layer of the ODS-MS/Al multilayer film. The adsorbed SDS molecules can be removed from the ODS-MS layer by flowing the water/ethanol mixture without SDS (Fig. 7(b), red arrow), and the coupling wavelength is restored to the original wavelength observed without flowing SDS.

In a study on the OWG mode using a porous anodic alumina as a waveguide layer on an $\mathrm{Al}$ thin layer, ${ }^{17}$ we reported that the shift of the coupling wavelength $\left(\Delta \lambda_{\mathrm{owG}}\right)$ was proportional to the total amount of an adsorbate on the top outer surface and on the inner pore surface of a porous waveguide layer. In the ODS-MS layer, since the area of the inner pore surface is much larger than that of the top outer surface, the SDS amount adsorbed on the top outer surface is negligibly small compared to that adsorbed on the inner pore surface, and $\Delta \lambda_{\text {OWG }}$ can be described as follows:

$$
\Delta \lambda_{\mathrm{OWG}}=a \cdot Q_{\mathrm{ads}}
$$

where $Q_{\text {ads }}$ and $a$ are the amount of SDS adsorbed on the inner pore surface and a proportional constant, respectively. The proportional constant, $a$, is mainly determined by the porosity and the surface area of the ODS-MS layer and the refractive indices of the ODS-MS layer and the SDS adlayer. Therefore, the time-course shift of the coupling wavelength shown in Fig. 7(b) represents the adsorption kinetics of SDS on the inner pore surface of the ODS-MS layer.

From the adsorption isotherms of SDS on the ODS-MS layer (inset of Fig. 7(b)), it is confirmed that the adsorption becomes saturated under a continuous flow of the SDS solution containing more than $0.5 \mathrm{mM}$ of SDS. This adsorption behavior depending on the bulk SDS concentration is comparable with the results reported for the adsorption isotherms of SDS on ODS-modified porous silica (ODS Hypersil). ${ }^{30}$

For adsorption at a thin porous film, the time-dependent amount of an adsorbate that diffuses into a porous layer is described by the following equation of an exponential series: ${ }^{31,32}$

$$
\frac{Q_{\text {ads }}(t)}{Q_{\text {ads }}(\infty)}=1-\frac{8}{\pi^{2}} \sum_{n=0}^{\infty} \frac{\exp \left[-\pi^{2}(2 n+1)^{2} D_{\text {app }} t / l^{2}\right]}{(2 n+1)^{2}}
$$

where $Q_{\text {ads }}(t), Q_{\text {ads }}(\infty), D_{\text {app }}$, and $l$ are the total amount of the adsorbate within the porous layer at time $t$, the amount of the adsorbate with equilibrium, the apparent diffusion coefficient (in $\mathrm{cm}^{2} \mathrm{~s}^{-1}$ ), and the thickness of the porous layer (in $\mathrm{cm}$ ), respectively. Since $\Delta \lambda_{\text {owg }}$ is proportional to the amount of SDS within the ODS-MS layer (Eq. (1)), Eq. (2) can be rewritten as

$$
\Delta \lambda_{\text {owG }}(t)=\Delta \lambda_{\text {owG }}(\infty)\left(1-\frac{8}{\pi^{2}} \sum_{\mathrm{n}=0}^{\infty} \frac{\exp \left[-\pi^{2}(2 n+1)^{2} D_{\text {app }} t / l^{2}\right]}{(2 n+1)^{2}}\right)(3)
$$

We used Eq. (3) to examine the adsorption kinetics of SDS at the ODS layer of the ODS-MS/Al multilayer film. The critical micellar concentration $(\mathrm{cmc})$ of SDS in the water/ethanol mixture solution $(99: 1 \mathrm{v} / \mathrm{v}, 0.4 \mathrm{M} \mathrm{NaCl}$ :ethanol) was estimated as $0.25 \mathrm{mM}$ by fluorescence measurements using Rhodamine $6 \mathrm{G}$ according to the literature. ${ }^{33}$ The adsorption data were obtained under a flow of a SDS solution with the concentration of $5 \mu \mathrm{M}$, which is much lower than cmc, and it was considered that SDS was dispersed in the solution without significant aggregation.

Figure 8 shows the adsorption kinetic data and their best-fit result to Eq. (3). The fitting analysis provides the apparent diffusion coefficient of $5.3 \times 10^{-13} \mathrm{~cm}^{2} \mathrm{~s}^{-1}$, although the fitting curve slightly deviates from the measured data. The deviation would be ascribed to the pore geometry of the ODS-MS layer. Equation (2) assumes that a molecule diffuses in a porous film with a straight cylindrical pore geometry without tortuosity or constrictions,${ }^{32}$ while the ODS-MS film with a layered structure was used in the present study. In addition, hydrophobic interaction between the dodecyl group of SDS and the octadecyl group of the surface ODS would also contribute to the deviation, because the deviation tends to increase for strong interactions 


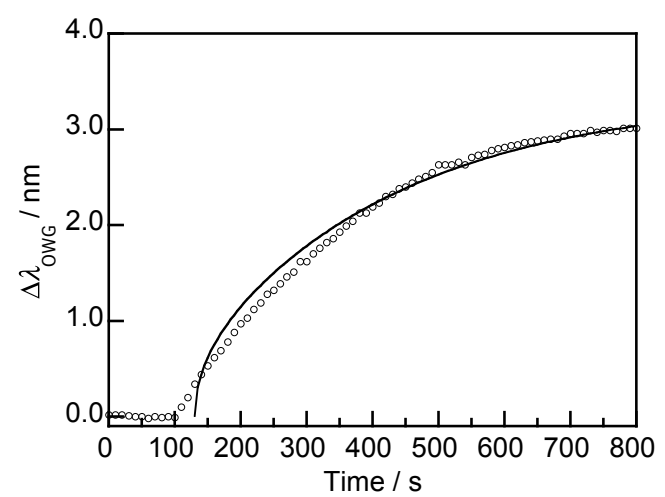

Fig. 8 Adsorption dynamics of SDS under the flow of $5 \mu \mathrm{M}$ solution. Plots are experimental data and the solid line is the result by best fit using Eq. (3).

between the diffusing molecules and the inner pore surface. ${ }^{32}$ Despite the slight deviation, the order of the apparent diffusion coefficient is considered to be reasonable because the same order of the apparent diffusion coefficient $\left(2.4 \times 10^{-13} \mathrm{~cm}^{2} \mathrm{~s}^{-1}\right)$ was reported in the desorption study of Rhodamine $6 \mathrm{G}$ within an MS sphere. ${ }^{7}$ The results obtained in the present study suggest that OWG spectroscopy based on the MS/Al multilayer film is useful to discuss dynamic processes occurring in MS materials.

\section{Conclusion}

In this study, the ODS-modified mesoporous silica on the $\mathrm{Al}$ film (ODS-MS/Al) was fabricated. Its characteristics and the adsorption behavior of SDS in ODS-MS were examined by using OWG spectroscopy. The ODS-MS layer fabricated in this study had a layered type structure with open accessible pores (pore diameter, ca. $6 \mathrm{~nm}$ ) on its surface. The ODS-MS/Al multilayer film showed a clear waveguide coupling dip under the flow of an ethanol solution and a water/ethanol mixture, whereas no waveguide coupling dip was observed under the flow of a water solution. When water was flowed, the refractive index of the ODS-MS layer was lower than that of bulk water, since water molecules could not penetrate into the hydrophobic ODS-modified silica mesopores. Under the flow of ethanol or a water/ethanol mixture (99:1 v/v), the mesopores of the ODS-MS layer are filled with ethanol and/or water molecules, and the refractive index of the ODS-MS layer becomes higher than that of the bulk solution phase. As a result, clear waveguide coupling dip can be observed under the flow of ethanol or the water/ethanol mixture. When the water/ethanol mixture containing SDS was flowed, the ODS-MS/Al multilayer film showed the shift of the waveguide coupling dip corresponding to adsorption/desorption of SDS. From the time-course shift of the waveguide coupling dip, the adsorption isotherm and the diffusivity of SDS inside the mesopores could be obtained. These results indicate the usefulness of OWG spectroscopy for the study of adsorption/desorption dynamics in MS materials.

\section{Acknowledgements}

This work was supported in part by Grants-in-Aid for Scientific Research (Nos. 21685009 and 22225003) from the Ministry of Education, Culture, Sports, Science and Technology, Japan.

\section{References}

1. (a) C. Sanchez, C. Boissiere, D. Grosso, C. Laberty, and L. Nicole, Chem. Mater., 2008, 20, 682. (b) A. Vinu, K. Z. Hossain, and K. Ariga, J. Nanosci. Nanotechnol., 2005, 5, 347. (c) B. Hatton, K. Landskron, W. Whitnall, D. Perovic, and G. A. Ozin, Acc. Chem. Res., 2005, 38, 305. (d) H. Yoshitake, New J. Chem., 2005, 29, 1107. (e) G. J. A. A. Soler-Illia, C. Sanchez, B. Lebeau, and J. Patarin, Chem. Rev., 2002, 102, 4093.

2. (a) A. Yamaguchi, F. Uejo, T. Yoda, T. Uchida, Y. Tanamura, T. Yamashita, and N. Teramae, Nat. Mater., 2004, 3, 337. (b) A. Yamaguchi, H. Kaneda, W. Fu, and N. Teramae, $A d v$. Mater., 2008, 20, 1034. (c) A. Yamaguchi, M. M. Mekawy, Y. Chen, S. Suzuki, K. Morita, and N. Teramae, J. Phys. Chem. B, 2008, 112, 2024. (d) A. Yamaguchi and N. Teramae, Anal. Sci., 2008, 24, 25.

3. (a) W. Fu, A. Yamaguchi, H. Kaneda, and N. Teramae, Chem. Commun., 2008, 853. (b) T. Itoh, R. Ishii, T. Hanaoka, Y. Hasegawa, J. Mizuguchi, T. Shiomi, T. Shimomura, A. Yamaguchi, H. Kaneda, N. Teramae, and F. Mizukami, J. Mol. Catal. B, 2009, 57, 183.

4. V. S.-Y. Lin, C.-Y. Lai, J. Huang, S.-A. Song, and S. Xu, J. Am. Chem. Soc., 2001, 123, 11510.

5. Q. Ji, M. Miyahara, J. P. Hill, S. Acharya, A. Vinu, S. B. Yoon, J. S. Yu, K. Sakamoto, and K. Ariga, J. Am. Chem. Soc., 2008, 130, 2376.

6. P. Schon, R. Michalek, and L. Walder, Anal. Chem., 1999, 71,3305 .

7. J. B. S. Ng, P. Kamali-Zare, H. Brismar, and L. Bergstrom, Langmuir, 2008, 24, 11096.

8. K. Nakatani and T. Sekine, Langmuir, 2000, 16, 9256.

9. K.-S. Mun, S. D. Alvarez, W.-Y. Choi, and J. Sailor, ACS Nano, 2010, 4, 2070.

10. Z.-M. Qi, I. Honma, and H. Zhou, Anal. Chem., 2006, 78, 1034.

11. H. Ouyang, L. A. DeLouise, B. L. Miller, and P. M. Fauchet, Anal. Chem., 2007, 79, 1502.

12. F. Cunin, T. A. Schmedake, J. R. Link, Y. Y. Li, J. Koh, S. N. Bhatia, and M. J. Sailor, Nat. Mater., 2002, 1, 39.

13. Z.-M. Qi, I. Honma, and H. Zhou, Appl. Phys. Lett., 2007, 90, 011102.

14. P. J. Cameron, A. T. A. Jenkins, W. Knoll, F. Marken, E. V. Milsom, and T. L. Williams, J. Mater. Chem., 2008, 18, 4304.

15. K. H. A. Lau, L.-S. Tan, K. Tamada, M. S. Sander, and W. Knoll, J. Phys. Chem. B, 2004, 108, 10812.

16. A. Yamaguchi, K. Hotta, and N. Teramae, Anal. Chem., 2009, 81, 105.

17. K. Hotta, A. Yamaguchi, and N. Teramae, Anal. Chem., 2010, 82, 6066.

18. W. Knoll, Annu. Rev. Phys. Chem., 1998, 49, 569.

19. S. Devanathan, Z. Salamon, G. Tollin, J. C. Fitch, T. E. Meyer, E. A. Berry, and M. A. Cusanovich, Biochemistry, 2007, 46, 7138.

20. J. S. Beck, J. C. Vartuli, W. J. Roth, M. E. Leonowicz, C. T. Kresge, K. D. Schmitt, C. T.-W. Chu, D. H. Olson, E. W. Sheppard, S. B. McCullen, J. B. Higgins, and J. L. Schlenker, J. Am. Chem. Soc., 1992, 114, 10834.

21. S. Inagaki, Y. Fukushima, and K. Kuroda, J. Chem. Soc. Chem. Commun., 1993, 680.

22. H. Nishihara, Y. Fukura, K. Inde, K. Tsuji, M. Takeuchi, and T. Kyotani, Carbon, 2008, 46, 48.

23. D. R. Dunphy, S. Singer, A. W. Cook, B. Smarsly, D. A. 
Doshi, and C. J. Brinker, Langmuir, 2003, 19, 10403.

24. T. Yokoyama, R. Ishii, T. Itoh, K. Kitahata, S. Matsuura, T. Tsunoda, S. Hamakawa, T. Hanaoka, H. Nanbu, and F. Mizukami, Mater. Lett., 2011, 65, 67.

25. K. A. Koyano, T. Tatsumi, Y. Tanaka, and S. Nakata, J. Phys. Chem. B, 1997, 101, 9436.

26. S. P. Naik, S. Yamakita, M. Ogura, and T. Okubo, Microporous Mesoporous Mater., 2004, 75, 51.

27. V. Antochshuk and M. Jaroniec, Chem. Mater., 2000, 12 , 2496.

28. A. Yamaguchi, T. Yoda, S. Suzuki, K. Morita, and N.
Teramae, Anal. Sci., 2006, 22, 1501.

29. D. F. Rohlfing, J. Rathousky, Y. Rohlfing, O. Bartels, and M. Wark, Langmuir, 2005, 21, 11320.

30. A. Berthod, I. Girard, and C. Gonnet, Anal. Chem., 1986, $58,1356$.

31. J. Crank, "Mathematics of Diffusion", 2nd ed., 1975, Claredon Press, Oxford.

32. D. Rivera and J. M. Harris, Anal. Chem., 2001, 73, 411.

33. J. C. Micheau, G. V. Zakharova, and A. K. Chibisov, Phys. Chem. Chem. Phys., 2004, 6, 2420. 